\section{Correction of Measurements of Low-Angle X-Ray Scattering}

IT has been recognized that for accurate measurements of small-angle scattering it is necessary to use a monochromator. The monochromatized beam may have a very small width, but has a finite height which cannot be reduced without making the intensity of the beam too weak. If the intensity scattered at angle $\varepsilon$ is $i(\varepsilon)$, experiment does not give directly $i(\varepsilon)$, but $I(\varepsilon)=2 \int_{0}^{A} i\left(\sqrt{\varepsilon^{2}+\frac{t^{2}}{D^{2}}}\right) d t$; where $A$ is the halfheight of the beam, $D$ the distance between sample and film, and $t$ the distance between one point of the beam and the plane of symmetry. For any value of $A$, the difference between $i(\varepsilon)$ and $I(\varepsilon)$ becomes important below a given value of $\varepsilon$. If $I(\varepsilon)$ is practically zero for $\varepsilon>\varepsilon_{0}$ and if $A>D \varepsilon_{0}, I(\varepsilon)$ may be written more simply:

$$
I(\varepsilon)=2 \int_{0}^{\infty} i\left(\sqrt{\varepsilon^{2}+\frac{t^{2}}{D^{2}}}\right) d t .
$$

The approximate formula for $i^{1}$ is

$$
i(\varepsilon)=\text { const. } \exp \left(\frac{-4 \pi^{2} R^{2} \varepsilon^{2}}{3 \lambda^{2}}\right),
$$

where $R$ is the radius of gyration of the diffracting particles and $\lambda$ the wave-length of the incident radiation. If this particular function is put in formula (1), it is found ${ }^{2}$ that :

$$
I(\varepsilon)=\frac{\sqrt{3}}{4 \sqrt{\pi}} \cdot \frac{\lambda D}{R} i(\varepsilon) .
$$

But formula (2) is only valid for small values of $\varepsilon$, and the integral (1) involves the whole exponential function. Thus, the result (3) is not correct.

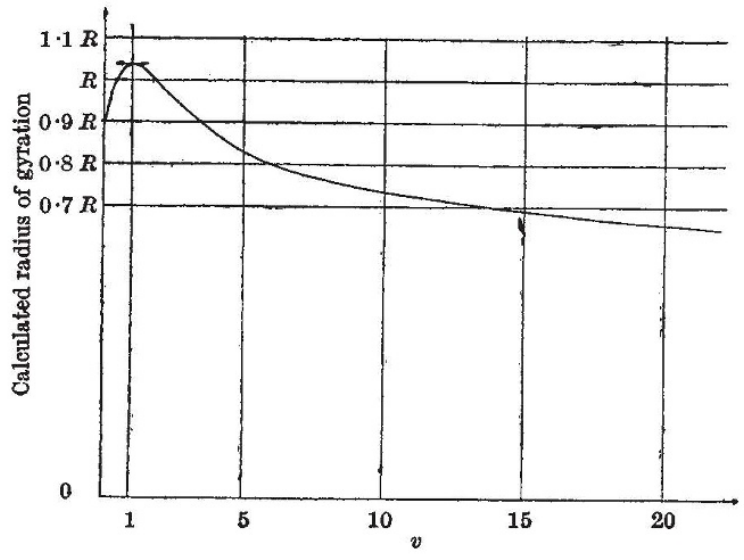

Fig. 1

We have calculated the rigorous function $i(\varepsilon)$ for spherical and ellipsoidal (axial ratio $=v$ ) particles of the same radius of gyration $R$. Then we calculated the corresponding functions $I(\varepsilon)$. The result is that the approximate exponential form for $I(\varepsilon)$ is not the same as for $i(\varepsilon)$; the curve (Fig. 1) gives the calculated radius of gyration from $I(\varepsilon)$ according to (3), and shows that the error is $\mathbf{3 . 5}$ per cent for spherical particles but grows very large for plateshaped and particularly needle-shaped particles. It can be shown, also, that the error is important in the case of mixtures of almost spherical particles of various diameters.

To obtain the corrected function $i(\varepsilon)$, Shull and Roess $^{3}$ decomposed the experimental function $I$ into a sum of exponentials :

$$
I=\Sigma_{m} C_{m} \exp \left(-m^{2} \varepsilon^{2}\right) .
$$

Then, according to (1),

$$
i=\Sigma \frac{m C_{m}}{\sqrt{\pi} D} \exp \left(-m^{2} \varepsilon^{2}\right) .
$$

But this decomposition is not possible in every case, for example, for patterns with rings of very small diameter 4 .

There exists a general method of solving equation (1) (the calculation is due to MM. Bouzitat and Germain, of the Office National de Recherches Aéronautiques). The result is as follows :

$$
i(\varepsilon)=-\frac{1}{\pi D^{2}} \int_{0}^{\infty} \frac{I^{\prime}\left(\sqrt{\left.\varepsilon^{2}+\frac{u^{2}}{D^{2}}\right)}\right.}{\sqrt{\varepsilon^{2}+\frac{u^{2}}{D^{2}}}} d u ;
$$

( $u$ is the integration parameter).



To use (4), it is necessary to determine the derivative $I^{\prime}(\varepsilon)$ from the experimental data. In order to check the accuracy of the method in a practical case, we considered the case of a schematic ring (Fig. 2 a), and we calculated the curve which would be observed with a beam of infinite height (Fig. $2 b$ ). Then we determined graphically $i(\varepsilon)$ from curve $2 b$ by means of the equation (4). The curve obtained (Fig. $2 \mathrm{c}$ ) is in satisfactory agreement with the theoretical curve.

\section{A. GUTNIER \\ G. Fournet}

Laboratoire d'Essais du Conservatoire National des Arts et Métiers,

Office National de Recherches Aéronautiques,

$$
\text { Paris. }
$$

1 Guinier, A., Ann. Phys., 12, 192 (1939).

- Hoseman, Z. Elektrochem., 46, 535 (1940).

Shull, C. G., and Roess, L. C., J. Appl. Phys., 18, 304 (1947).

- Riley, D. P., "The Ultra-Fine Structure of Coals and Cokes", Brit. Coal Util. Res. Assoc., 232 (London, 1944). Guinier, A., J. 'Chim. Phys., 40, 133 (1943). 